\title{
Design of an inherently safe Worm-like Robot
}

\author{
Martin Eder, Maximilian Karl, Felix Schultheiß, \\ Johannes Schürmann and Alois Knoll \\ Faculty of Informatics, Robotics and Embedded Systems \\ Technische Universität München \\ D-85748 Garching, Germany \\ Email: ederma@in.tum.de
}

\author{
Stefan Riesner \\ Robotics Technology Leaders GmbH \\ D-81249 München, Germany \\ Email: s.riesner@rtleaders.com
}

\begin{abstract}
Nowadays robots are disseminated more and more in fields where humans are in the loop. These collaborative modes are always characterized by safety issues, in particular problems regarding compliance in case of contact. Our approach to solve these problems is based on passive compliance, which means that an inherently flexible robotic mechanism is designed driven by pneumatic artificial muscles (PAM). Compared to state of the art robots the novelty here is a completely modular and decentralized setup in terms of both mechanical and control architecture. Main benefit of this new design is expandability and increased precision. This paper presents the design and control of a robot of this kind with 3 segments or rather 6 degrees of freedom (DOF), which proves the novel concept.
\end{abstract}

\section{INTRODUCTION}

In industrial environment robots are normally surrounded with safety fences to securely separate human and machine. In accordance with currently valid regulations, robotic arms used for human-robot interaction mustn't exceed an overall end effector velocity of $250 \mathrm{~mm} / \mathrm{s}$. However, this way of proceeding is not always practicable for robots within collaborative applications. New regulations such as the ISO/TS $15066^{1}$ or the prEN ISO $13482^{2}$ are in the pipeline. These new norms try to cover robots in the field of human-robot interaction.

Most important question related to safe human-robot interaction is dealing with adequate compliance of the system. Two different approaches to come up with compliance are prevalent: active and passive compliance. Whereas active compliance is dependent on force or rather force-torque sensors that influence the robots behavior on a control level, passive compliance is based on a flexible structure of the robot. Since passive compliance doesn't need expensive sensor integration, inherently compliant systems are economically advantageous. Also passive compliance has the advantage that even in case of control failure flexible properties are guaranteed.

\section{A. State of the Art}

Various robotic setups are already developed with passive or inherently compliant behavior. The already commercially available robot ROMO built by FerRobotics Compliant Robot Technology GmbH, Austria, is a flexible arm based on pneumatic artificial muscles. It has 3 connected levers with 3 parallel PAM each [1] with 5 DOF in total, i. e. 2 ball-andsocket joints with 2 DOF each and one cylindrical joint with

\footnotetext{
${ }^{1}$ robots and robotic devices - collaborative industrial robots

${ }^{2}$ robots and robotic devices - safety requirements for non-industrial robots - non-medical personal care robot
}

1 DOF. The reach of the arm is $2000 \mathrm{~mm}$, its payload is $5 \mathrm{~kg}$. Another compliant robotic system using PAM is the "Actuator system comprising an artificial air muscle" by Shadow Robot Company Ltd., London, UK [2]. It is an anthropomorphic robotic system or rather a hand-arm system with very ductile PAM. Beyond, the Bionic Handling Assistant (BHA) built by Festo AG \& Co. KG. is among the most crucial state of the art robots of this kind [3]. This robot is based on a hybrid serialparallel kinematics where three segments are arranged serially, each with parallel pneumatically inflated chambers, which is an operating principle that deals with expansion rather than contraction. Compared to its weight of $1.8 \mathrm{~kg}$ the payload of $0.5 \mathrm{~kg}$ with a reach of $1.2 \mathrm{~m}$ is rather high [4]. Finally, two more robotic demonstrators should be mentioned. The first one is presented in [5], which has 2 joints driven by 4 PAM in total. This planar setup was built by the Institute of Automation and Systems Engineering TU Ilmenau, Germany, in cooperation with Festo AG \& Co. KG. The second demonstrator also comes from the same institute, having 4 joints with 12 PAM, thus this setup is not limited to planar movements [6].

\section{B. Limitations and Solutions}

All of the aforementioned pneumatic robotic systems are characterized in that the control hardware is located in a central basis, which is also the base of the specific robot. As long as the segments are not too large in length and only a few segments are arranged serially, this approach is technically well feasible. But with increasing number of segments and segment length, also the number and length of supply lines escalates. In addition, the control accuracy decreases as the robots becomes longer, because the huge distance between control valves and actuators affects dead volume and less control precision as a result. With more segments, the arrangement of both supply lines and control wires is getting more complicated.

This problem can be solved if each of the robot's segments has its valve cluster and controller unit onboard. Such a decentralised setup leads to a very flexible and modular robot. Each unit of the mechanism works autonomously. Only one medium supply tube for feeding the segments with pressurized air is necessary together with one control bus line including power supply line [7].

\section{Organization of this Paper}

The outline of the paper is organized in the following way. In section II the structure, kinematics, dynamics as well as the controller are concerned in detail. In particular, the Robot 


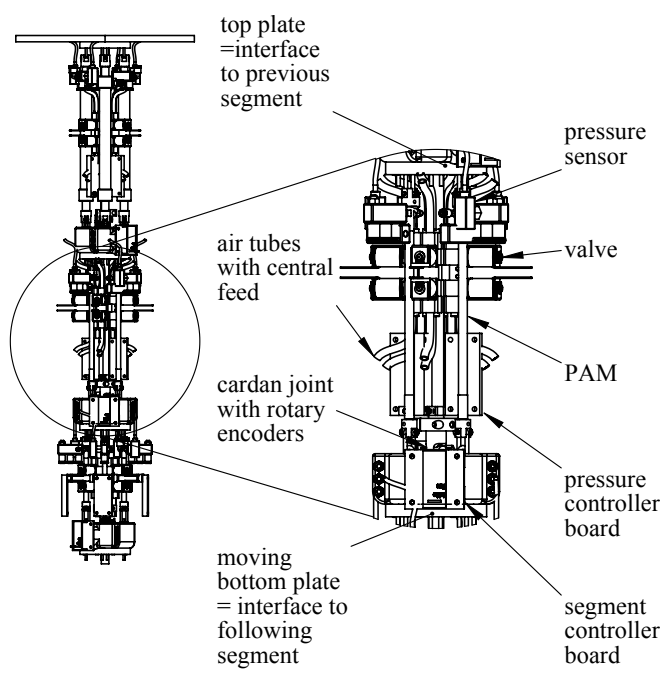

Fig. 1. Modular segment arrangement of Worm-like Robot

Control Subsection II-B highlights the adapted calibration method, the overall cascaded control strategy together with a calibration enhancement. Movement tests conducted to prove the concept as stated in the sections before are considered in section III, which is followed by conclusions section IV.

The specific contribution within this paper is the prototypic development of a novel inherently compliant pneumatic robot that is based on decentral control on the one hand and centralized medium feed on the other hand. Due to the local arrangement of all control components including valves and sensors on each segment, the setup is completely modular and scalable. The crucial advantage here is the increase in positioning accuracy. Due to the flexible character of the robot, safe human-robot interaction can be guaranteed.

\section{3-SEgments Robot Prototype}

In this paper a robot prototype with a total weight of less than $5 \mathrm{~kg}$ and an overall length of $800 \mathrm{~mm}$ is designed that is inherently compliant and thus safe in terms of human-robot interaction. The following paragraphs introduce the kinematics and dynamics as well as the control of the robot.

The overall structure of the robot is completely modular. 3 segments are driven by 12 PAM (Festo DMSP-10-160N) to generate 6 DOF. Two neighboring segments are interconnected by means of cardan joints offering two DOF, each actuated by a set of four PAM in antagonistic arrangement. Fig. 1 depicts an arrangement with 3 modular units of the robot with one segment highlighted. The novelty here is that each segment has its own controller and valve cluster.

\section{A. Kinematics and dynamics}

The forward kinematics of our worm-like robot is quite a straightforward problem due to the serial arrangement of axes, all of which are rotational cardan joints. Both joints of one cardan joint are considered as dual-axes with a common intersection point. The homogenous transformation from one frame to the subsequent frame according to DenavitHartenberg convention [8] yields:
TABLE I. DENAVIT-HARTENBERg (DH) PARAMETERS

\begin{tabular}{lcccc}
\hline Joint & $a_{i-1}$ & $\alpha_{i-1}$ & $d_{i}$ & $\theta_{i}$ \\
\hline 1 & $l_{0}$ & $-\frac{\pi}{4}$ & 0 & $\theta_{1}$ \\
2 & 0 & $\frac{\pi}{2}$ & 0 & $\theta_{2}$ \\
3 & $l_{1}$ & $-\frac{\pi}{4}$ & 0 & $\theta_{3}$ \\
4 & 0 & $\frac{\pi}{2}$ & 0 & $\theta_{4}$ \\
5 & $l_{2}$ & $-\frac{\pi}{4}$ & 0 & $\theta_{5}$ \\
6 & 0 & $\frac{\pi}{2}$ & 0 & $\theta_{6}$ \\
\hline
\end{tabular}

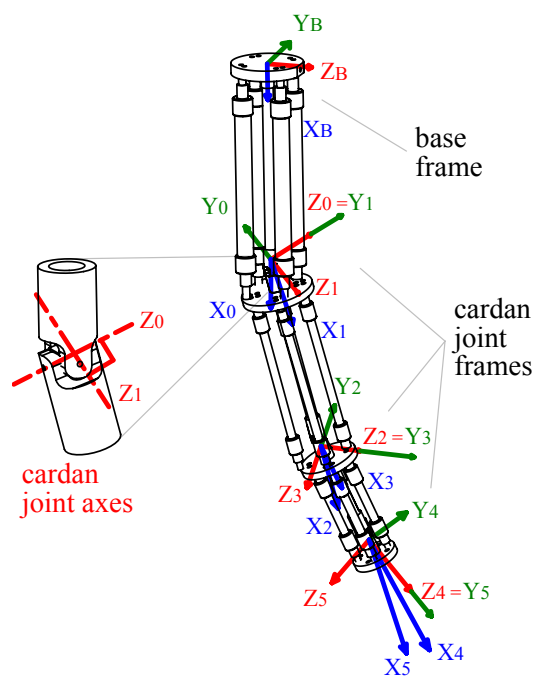

Fig. 2. Coordinate frames according to DH convention

$$
{ }_{i-1} T_{i}=\left(\begin{array}{cccc}
c \theta_{i} & -s \theta_{i} & 0 & a_{i-1} \\
s \theta_{i} c \alpha_{i-1} & c \theta_{i} c \alpha_{i-1} & -s \alpha_{i-1} & -s \alpha_{i-1} d_{i} \\
s \theta_{i} s \alpha_{i-1} & c \theta_{i} s \alpha_{i-1} & c \alpha_{i-1} & c \alpha_{i-1} d_{i} \\
0 & 0 & 0 & 1
\end{array}\right)
$$

This transform is based on the Denavit-Hartenberg (DH) parameters given in Tab. I. $l_{0}, l_{1}$ and $l_{2}$ represent the length of each segment measured along the structure from one cardan joint to the next one. The frames are depicted in Fig. 2.

Using DH parameters from Tab. I the expressions in Eq. 1 can be arranged within a complete transformation between the serial connection of frames as follows:

$$
{ }_{0} T_{n}={ }_{0} T_{11} T_{2} \ldots{ }_{4} T_{5}
$$

With Eq. 2 the forward kinematics is already solved.

For the current setup, the workspace of the robot is illustrated in Fig. 3. A 2D projection of the 3D workspace, which is rotational symmetric (in the first approximation) about the vertical axis is highlighted in violet. Its shape appears a little similar to a saucer. The maximum stroke of the worm-like mechanism is about $100 \mathrm{~mm}$, its total angular movement ability is $\pm 22 \mathrm{deg}$. The extremal configuration of robot is indicated in blue. In this extreme position, all angles of all the cardan joints are at maximum deflection, which is 16 deg each, which means that the entire setups curvature is greatest.

In order to calculate the forces and torques of the system based on corresponding motion conditions, in a first step the Newton-Euler method [9], [10], [11] is applied according to the calculation specification in projective manner: 


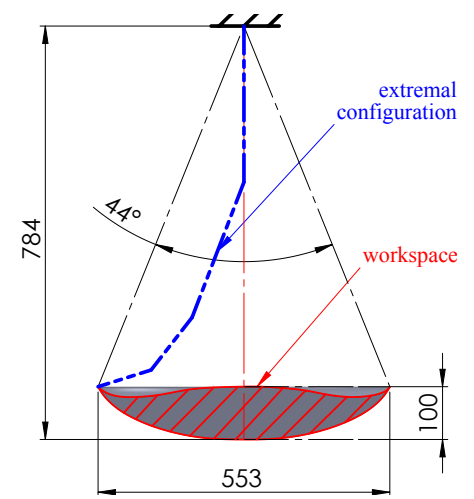

Fig. 3. Rotational symmetric workspace (2D projection)

$$
\sum_{i=1}^{3}\left[J_{T, i}^{T}\left(\dot{p}_{i}-F_{e, i}\right)+J_{R, i}^{T}\left(\dot{L}_{i}-M_{e, i}\right)\right]=0
$$

which has the following components: $i$ being $i^{\text {th }}$ body, $J_{T, i}=\frac{\partial \dot{r}_{0} s}{\partial \dot{q}}$ being the Jacobian of translation, $\dot{p}_{i}=m_{i} \ddot{r}_{0 s, i}$ being the momentum change, $F_{e, i}$ being active forces, $J_{R, i}=$ $\frac{\partial \omega_{0 s}}{\partial \dot{q}}$ being the Jacobian of rotation, $\dot{L}_{i}$ being the change in angular momentum, $M_{e, i}$ being the active torques or transversal momentum.

However, the representation in Eq. 3 is not practical if forces and torques are to be calculated for a given position or acceleration $(\ddot{q}(\dot{q}, q))$. Thus, in a second step the general motion equation that separates $\ddot{q}$ becomes

$$
M(q) \ddot{q}+h(q, \dot{q})=Q_{u}
$$

with mass matrix $M$ and $h$ being the vector of all forces excluding $Q_{u}$, which represent the driving forces/torques.

These dynamic relationships can be utilized to enhance the control that is presented in the following subsection II-B or rather in II-B1, which deals with the calibration of the PAMs, including force considerations in an initially static condition.

\section{B. Robot Control}

Our robot control is based on a cascaded pressure and position controller. For each PAM a customized pressure controller board based on Atmel ATTiny 24 microcontroller is utilized. Each pressure controller board has a current controller circuit with LM358D op-amp to manipulate the miniature proportional valves (Parker MDPro) whereas pressure data are coming from an analogue pressure sensor (SMC PSE 510). The outer position control loop integrates angle sensor data that are received from two rotary encoders (ams AS5145H) per cardan joint. These data are processed in superordinate segment controllers, which here are Arduino Nano 3.0 boards utilizing ATmega 328 microcontrollers. Both pressure controller boards and segment controller boards communicate by means of $i^{2} C$ bus.
1) Calibration Method: In order to have a correlation between the PAM pressure and the resulting angles of the setup, all of the muscles have to be calibrated before. The calibration of the system generates characteristics including information about the current pressure of the PAM, its length (kinematically convertible to contraction or joint angle, respectively) and the muscle force. In this manner the muscle force is important since the load of the PAM changes its length, therefore the loads due to external and internal gravity or inertial loads have to be considered. The calibration procedure here is an advantageous continuous development of [12]. Since the PAM are arranged in antagonistic way, the calibration is conducted pairwise, i.e. for each DOF separately and not for each PAM individually. Thus, calibration expenses decrease by $50 \%$. The weight of the subsequent segments is used in order to vary the forces that the PAM have to carry.

For each DOF, a set of 200 measurement data points are recorded. During each step, the pressure is set whereas the angular position is measured and the acting force is calculated. This procedure is done making use of a customized transformation tool that automatically projects the angular positions to corresponding muscle length and muscle force. In a next step the set of 200 normalized measurement data is then processed utilizing evolutionary symbolic regression via software tool Eureqa (creativemachines.cornell.edu/eureqa). The conversion is necessary in order to result in low cost functions that can be calculated easily with very little computing power provided by pressure board microcontrollers. The calibration steps for our robot with 3 segments or 6 DOF is as follows: (1) calibrate DOF i (set DOF !=i stiff); (2) set diff. pressures $p$ for PAM DOF i; (3.1) measure angle DOF i; (3.2) calculate forces DOF i using static equilibrium; (3.3) save pressure/angle/force for DOF i; (4) normalize and connect to eureqa; (5) create lowcost functions;

One example of how such a low cost function looks like for one PAM, can be given as follows:

$$
\begin{array}{r}
p_{01}(\alpha, f, c)=\frac{80.17 c-70.12 f}{184.59-64.99 \alpha+910.72 \alpha^{2}}+ \\
+0.04-0.67 \alpha+3.28 \alpha^{2}
\end{array}
$$

with the pressure $p_{01}$ of the PAM of segment 1 (statically attached to the ceiling) being dependent on the angle $\alpha$ of the corresponding joint, the muscle force $f$ and a stiffness value $c$, by means of which the stiffness of each joint can be adjusted. Correspondingly, other PAM actuators are characterized by similar functions, where the function constants are varied accordingly.

2) Cascaded Control Strategy: The control of this inherently safe robot pursues several objectives. As already introduced in II-B, first of all a decentralized architecture is chosen in order to guarantee modularity. Second, for the distributed controller devices control algorithms have to be implemented that can run on inexpensive small microcontrollers such as the ATTiny 24. Thus, a simplified modeling or calibration as introduced in subsection II-B1 is applied and integrated in the cascaded control scheme of Fig. 4. An innermost current control loop provides one current controller (CC) for adjusting the current $i_{i}$ of each of the valves (24 in total, both inlet and outlet), which is realized completely as a hardware solution. The next higher control loop is a pressure control loop with 


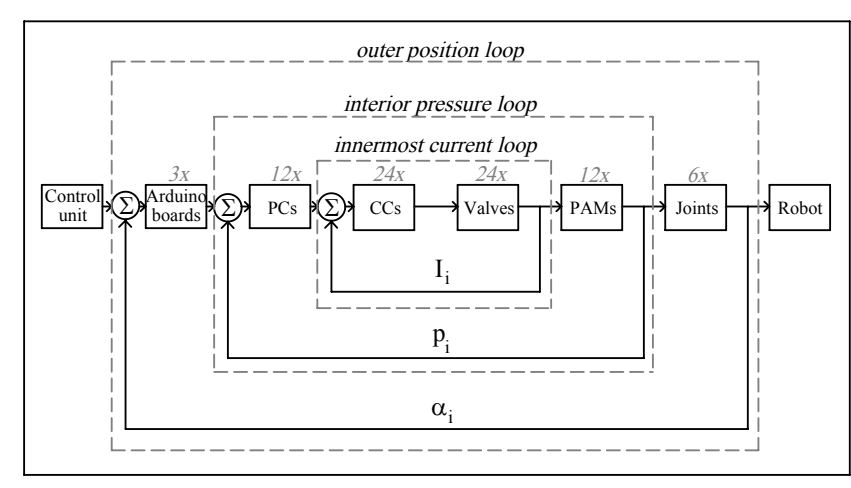

Fig. 4. Cascaded control scheme with current/pressure/position loops

one pressure controller board (PC) making use of a PID controller with Pulse Density Modulation (PDM) that regulates the pressure $p_{i}$ of each of the 12 PAMs. For this stage, the calibration characteristics in II-B1) that make a connection between pressures, forces and contraction or angles are utilized such that a pressure control corresponds with a force control. A third outermost control loop takes care about the position of the joints, i.e. controls joint angles $\alpha_{i}$ utilizing Arduino Nano boards. An external control unit is responsible for GUI and position setting purposes.

3) Control enhancement: In order to result in a pressure control loop as precise as possible, it is worth to carry out further research on the valve behavior, i.e. valve dynamics or pressure dynamics, which has direct influence on the quality of the control. Therefore, our Parker MDPro valves were fed with different pressures and different valve currents in ranges between $0-7$ bar or $160-300 \mathrm{~mA}$. As shown in the phase diagrams in Fig. 5 and 6, the change in pressure alters with both pressure and valve current. During the inflow cycle first the pressure change increases very fast until a first maximum is reached. Vice versa during the exhaust process, which has to be read from right to left in Fig. 6, the phase lines behave quite similar, but without the phenomenon of a second minimum. It was further found that both inlet and exhaust processes result in pretty similar flow rates or pressure changes, where inlet cycle is only slightly higher (about $3 \mathrm{bar} / \mathrm{s}$ ). This proves that the valve dimensions are properly dimensioned, i.e. the exhaust flow is only marginally lower. In some cases, the exhaust rates are extremely lower than the inlet rates, which makes the use of a second outlet valve necessary as described in [13].

In this paper it is claimed that the short stub lines that feed the PAMs of each segment and which are connected to a single central air feed tube have advantages in terms of response behavior of the PAMs since the dead volume between each valve and PAM becomes minimal. In order to estimate the effect of longer tubes between the valves and the actuators, pressure change tests according to Fig. 5 and 6 were conducted with different additional tubes. With these tests, a system similar to a centralized architecture approach can be achieved without completely rebuilding the entire robot. Tab. II illustrates the dependencies of the tube length compared to the pressure change rate over the pressure in average. The values from the phase diagrams in Fig. 5 and 6 are utilized as a reference $(100 \%)$.

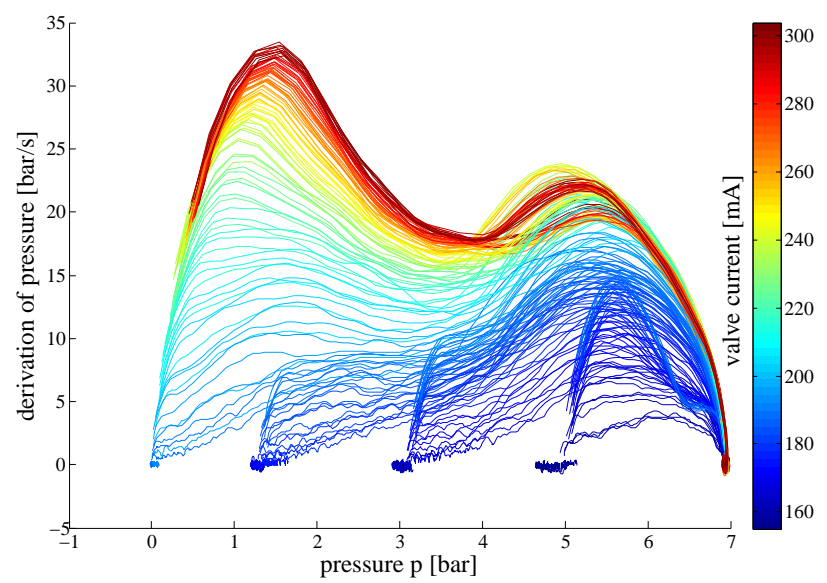

Fig. 5. Valve inlet phase diagram with pressure change rate

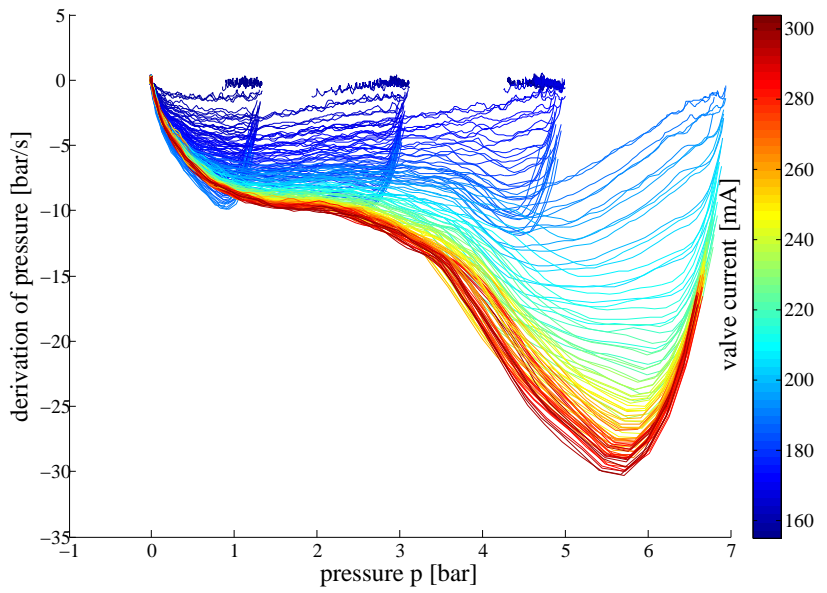

Fig. 6. Valve outlet phase diagram with pressure change rate

For further enhancing the control quality for the worm-like mechanism, the damping of the system which was not considered in the quasi-static calibration, is additionally regarded in what follows and thereby becomes part of the cascaded control theory. Tests by free oscillations of all robotic segments about the first joint of segment 1 with varying deflection were conducted while recording the joint angles $\phi$. In this manner, the logarithmic decrement $\Lambda$ based on $n$ full waves at different times $t_{k}$ or periods $T$ of a single PAM pair was measured:

$$
\Lambda=\frac{1}{n} \ln \left(\frac{\phi\left(t_{k}\right)}{\phi\left(t_{k}+n T\right)}\right)
$$

which can be transferred to a damping parameter $d$ with torsional stiffness $c_{T}$ and mass moment of inertia $\Theta$ :

$$
d=\frac{2 \Lambda \sqrt{c_{T} \Theta}}{\sqrt{4 \pi^{2}+\Lambda^{2}}}
$$

TABLE II. PRESSURE CHANGE RATE VS. TUBE LENGTH

\begin{tabular}{ll|ll}
\hline \multicolumn{2}{l|}{ tube length } & & $\dot{p}$ rate inlet / outlet \\
\hline (1) $0.1 m$ & (2) $1.1 m$ & (1) $100 \% / 100 \%$ & (2) $63 \% / 67 \%$ \\
(3) $2.1 m$ & (4) $3.1 m$ & (3) $46 \% / 49 \%$ & (4) $36 \% / 39 \%$ \\
(5) $4.1 m$ & (6) $5.1 m$ & (5) $29 \% / 33 \%$ & (6) $30 \% / 32 \%$ \\
\hline
\end{tabular}




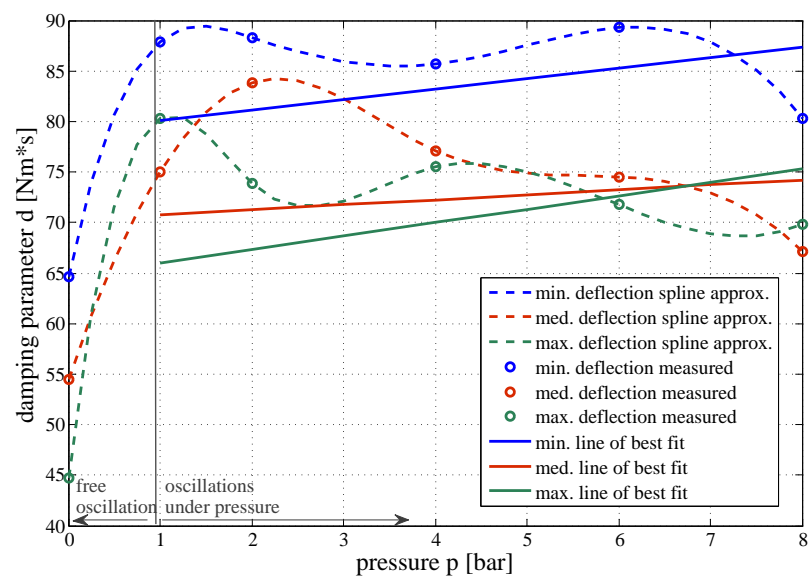

Fig. 7. PAM damping parameter vs. pressure

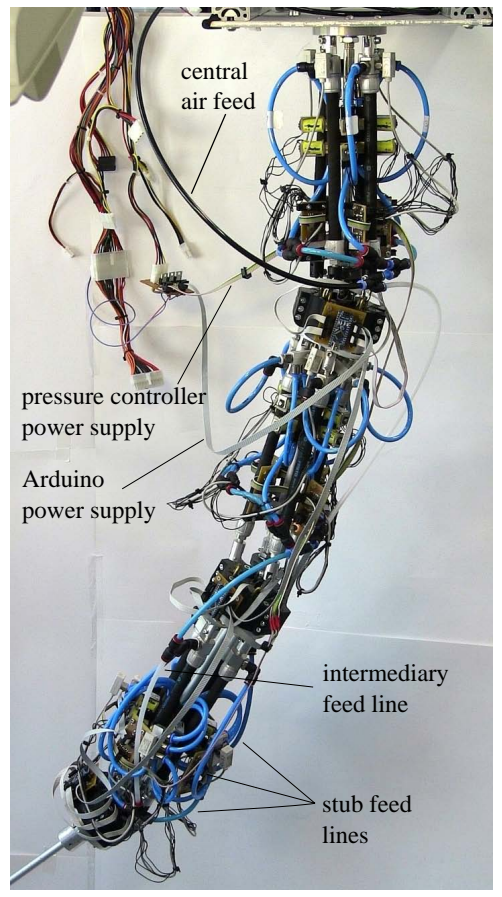

Fig. 8. Real Worm-like robot with 3 segments

This damping can be approximated to a correspondence as presented in Fig. 7, taking different angle of deflection into account (min.: 4.1deg, med.: 8.4deg and max.: 10.8deg).

\section{Movement Tests}

For testing the worm-like robot (cf. Fig. 8) several tests were conducted to prove its concept of a modular decentrally arranged human-friendly robot. It is claimed that the decentral placement of valves and the central air feed pipe has advantages regarding position accuracy since the lines between the valves and the PAM are kept extremely short, which should result in a better control behavior compared to a setup that has a central feed at the base.

Accordingly, the following experiments were conducted to prove the aforementioned statements: (1) circular movements of all segments: comparison of actual angles vs. set values; (2)
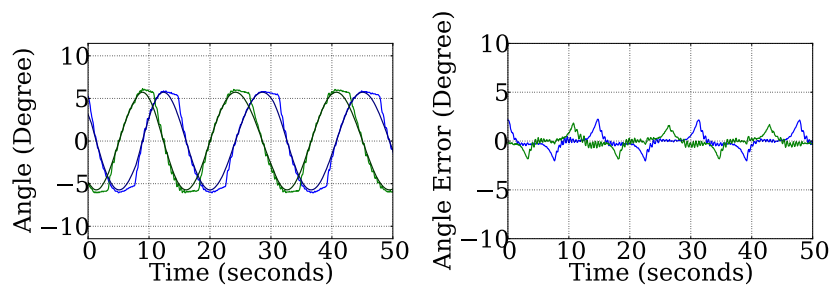

(a) motion of first segment
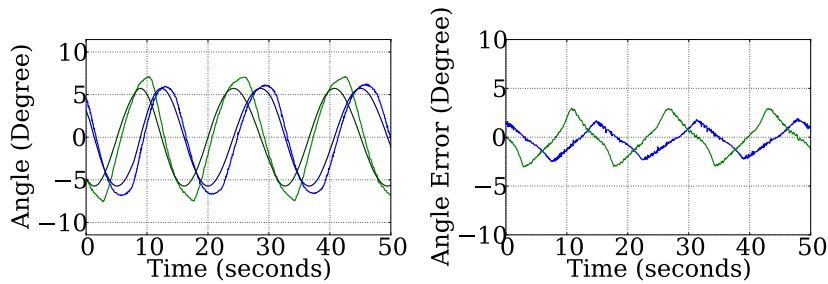

(b) motion of second segment
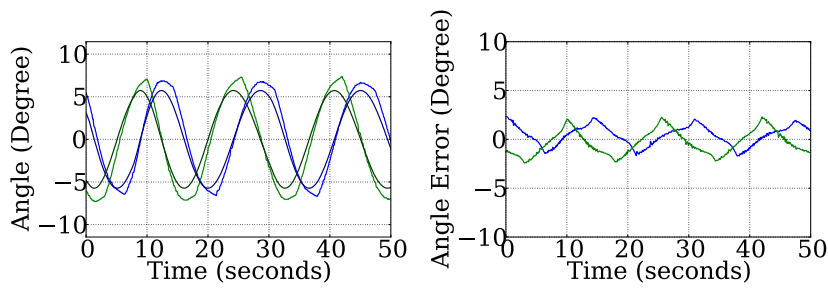

(c) motion of third segment

Fig. 9. Results of sinusoidal movement experiments

circular movements of all segments: increasing feed line length by $3 m$ as well as speed (multiplied by 3 ) and comparison of actual angles vs. set values; (3) measurement of joint torques. All experiments were run 5 times and results were averaged.

The test results for (1) are illustrated in Fig. 9. Angle measurements are based on data analysis of the rotary encoders that are attached to each of the joints, with a resolution of $12 \mathrm{Bit}$ and $1 \mathrm{M} \mathrm{Hz}$ readout frequency, transmitted to processing Arduino Nano boards via SSI protocol. All segments move in a circular way, which means that each of the joints rotates in a sinusoidal motion. For every single segment the set values (sine wave of first cardan joint axis colored in green, second one in blue) and the actual angles (first cardan joint axis colored in green, second one in blue) are depicted including their discrepancies. It can be seen that segment 1 (stationary fixed) has the minimum deviation of the measured and calculated angle values, which is easily comprehensible since the mutual influence of the moving segments 1,2 and 3 causes more oscillations for the subsequent segments 2 and 3 which are completely freely moveable.

Test results for (2) are shown in Tab. III, which depicts mean angle errors of different experimental configurations for all 3 segments. Here, the air feed tubes were extended from about $0.1 \mathrm{~cm}$ (short) by $3 \mathrm{~m}$ (long) and the joint speeds were varied between $(1 \mathrm{x}=1 \mathrm{deg} / \mathrm{s})$ and $(3 \mathrm{x}=3 \mathrm{deg} / \mathrm{s})$. This is done to prove the bad influence of additional tube length in terms of dead volume or control accuracy and to show the influence of control speed. In case of state of the art robots, the tube length could be within this range since nowadays all the systems use central control units that are located at a base. 
TABLE III. MEAN ANGLE ERRORS OF DIFFERENT CONFIGURATIONS [IN deg]

\begin{tabular}{llll}
\hline configuration & Seg. 1 & Seg. 2 & Seg. 3 \\
\hline short $(1 \mathrm{x})$ & 0.97 & 2.44 & 2.04 \\
short $(3 \mathrm{x})$ & 3.00 & 2.67 & 1.96 \\
long $(1 \mathrm{x})$ & 1.02 & 2.55 & 2.01 \\
long $(3 \mathrm{x})$ & 3.11 & 3.45 & 2.18 \\
\hline open loop short $(1 \mathrm{x})$ & 7.64 & 8.14 & 11.13 \\
\hline
\end{tabular}

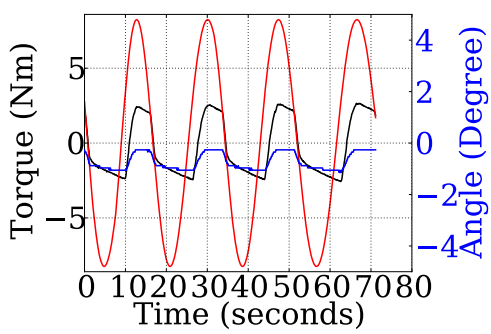

Fig. 10. Joint torques of one segment (sinusoidal movements)

The distance between the base and the actuators increases with the number of links the robot has. The results in Tab. III show evidence for an increase of angle errors with both speed and additional feed tube length, whereas the proximal segments (closer to the stationary basement) are influenced more than the distal segments. This is due to the fact that proximal segments have to cope with more inertia caused by subsequent segments, which leads to control problems using both longer tubes and higher velocity. For reference, angle errors are also given in open loop configuration with short air tubes at single speed.

To estimate the output forces/torques and thereby evaluate occuring impact forces/torques, a third experiment (3) determines joint torques. These tests were conducted utilizing a micro load cell (Phidgets CZL635) with a max. rated output error of $\pm 150 \mu \mathrm{V} / \mathrm{V}$ that is based on strain gauges, arranged with a Wheatstone Bridge (PhidgetBridge 1046) to measure shear forces at $8 \mathrm{~ms}$ data rate and 24 Bit differential voltage resolution. Test results are depicted in Fig. 10, which shows the actuating torque (black) of one joint while moving in a sinusoidal manner (red trajectory) with joint angles (blue). It can be seen that the torques or forces are not completely symmetrically with respect to the mechanism's longitudinal axes. The maximum joint torque measured during maximum deflection of a segment is approximately $6.3 \mathrm{Nm}$, whereas the maximum torque in not deflected state it is only $3.8 \mathrm{Nm}$, both applied with a maximum pressure of 6.9bar. Taking the kinematics of the robot into account, if the maximum torque value is applied to every joint, the system would be already safe for human-robot interaction if collision appears with back/shoulders, buttocks or thigh/knee with respect to the draft of ISO/TS 15066. In our case, the overall maximum impact forces are smaller than $200 \mathrm{~N}$ and the contact areas of all robot parts are sufficient to fall below the limits for pressure surface pressing. To guarantee safety for other body regions, the PAM pressure can easily be limited and the contact surfaces can be enlarged using an appropriate outer shell.

\section{CONCLUSIONS}

As it can be seen from the experimental results in the previous section III, an advantageous modular robot with quite high precision could be developed. The main advantage of the robot is the use of inherently compliant actuators, which make it a solution for safe human-robot interaction. Due to its arrangement as a series of independently controllable segments, the setup is scalable in terms of number of modules. Also the size of the modules can be varied utilizing different types of PAMs (length, diameter). Since the control of the robot is based on pressure control with subordinate consideration of forces or torques, an adjustment of output forces is easily realized. In this manner, the stiffness of the robot can be specified arbitrarily, which allows force limitation according to norms such as the upcoming ISO/TS 15066. Another benefit of the construction is the use of a single air feed line, which provides advantages particularly if many segments are attached to each other, because otherwise very many parallel feed lines would cause a large mechanism diameter.

Regarding future work, based on the current principles a continuum style robot is intended to be developed that allows enhanced maneuverability and reduces the number of rigid bodies, which increases safety for human-robot interaction.

\section{REFERENCES}

[1] P. Ferrara, "Robot Arm". Patent application, application number US2009/0182436A1, 2009

[2] M. P. Godden, R. M. Greenhill et al., "Actuation systems and artificial muscles therefor". Patent application, application number GB2385380A, 2003

[3] A. Grzesiak, R. Becker and Alexander Verl, "The Bionic Handling Assistant: a success story of additive manufacturing". In: Journal of Assembly Automation (2011), Vol. 31, issue. 4, pp. 329-333, Emerald Group Publishing Limited, 2011

[4] M. Rolf and J. J. Steil, "Constant curvature continuum kinematics as fast approximate model for the Bionic Handling Assistant". In: Proceedings of the International Conference on Intelligent Robots and Systems (IROS), Vilamoura, Algarve, Portugal, pp. 3440-3446, 2012

[5] A. Hildebrandt, O. Sawodny et al. "Cascaded control concept of a robot with two degrees of freedom driven by four artificial pneumatic muscle actuators". In Proceedings of the 2005 American Control Conference, Portland, USA, pp. 680-685, June 8-10, 2005

[6] T. T. Nguyen, C. Ament and M. Eichhorn, "A Nonlinear Control Design of the 4-Axis Kinematic Manipulator AirArm Driven by Pneumatic Muscle Actuators". In:Proceedings of the IEEE International Conference on Control and Automation 2009, Christchurch, New Zealand, pp. 23412347, December 2009

[7] A. Knoll. "Worm-like mechanism". U.S. Patent US 8,201,473 B2, June 19,2012

[8] J. Denavit and R. S. Hartenberg, "A Kinematic Notation for Lower-Pair Mechanisms Based on Matrices". In:Journal of Applied Mechanics, pp. 215-221, June 1955

[9] J. J. Craig, "Introduction to Robotics: Mechanics and Control". Pearson Education, Inc., Pearson Prentice Hall, 3rd edition, 2005

[10] M. Spong, S. Hutchinson and M. Vidyasagar, "Robot Modeling and Control". John Wiley \& Sons, Inc. 1st edition, 2005

[11] P. Corke, "Robotics, Vision and Control: Fundamental Algorithms in Matlab". Springer Tracts in Advanced Robotics, 2011

[12] M. Eder et al., "Compliant worm-like robotic mechanism with decentrally controlled pneumatic artificial muscles." In: Proceedings of the 2012 IEEE First International Conference on Innovative Engineering Systems, pp. 263-268. IEEE Press, 2012.

[13] D. Shin, I. Sardellitti and O. Khatibb. "A Hybrid Actuation Approach for Human-Friendly Robot Design". In:Proceedings of the IEEE International Conference on Robotics and Automation, pp. 1747-1752, Passadena, USA, May 2008 